\title{
Transmediastinal Trachea Closure after Dilational Tracheotomy Positioned Too Low Down
}

\author{
Andreas Kirschbaum ${ }^{1}$ Tanja Maier ${ }^{2}$ Afsin Teymoortash ${ }^{3}$ \\ ${ }^{1}$ Department of Surgery, University Hospital, Baldingerstrasse, \\ Marburg, Germany \\ 2 Department of Nephrology, University Hospital, Marburg, Germany \\ ${ }^{3}$ Ear, Nose and Throat, University Hospital, Marburg, Germany \\ Thorac Cardiovasc Surg Rep 2016;5:4-7. \\ Address for correspondence Andreas Kirschbaum, MD, Department \\ of Surgery, Baldingerstrasse, University Hospital, Marburg 35033, \\ Germany (e-mail: Kirschbaum001@gmx.de).
}

\begin{abstract}
Keywords

- airway

- trachea

- complications
\end{abstract}

\section{Background}

Tracheotomy should be considered for intensive care patients who are likely to need mechanical ventilation for a longer period. At present, most tracheotomies in intensive care units are performed at the bedside using percutaneous dilational tracheotomy (PDT). ${ }^{1}$ Various techniques are available. At this hospital, the Chiaglia Blue Rhino technique is the only one used. This involves puncturing the trachea at the level of the second to fourth cartilaginous ring under bronchoscopic visualization. A wire is advanced through the needle used for puncture. This wire serves as a guide for the bougie tube introducer that is used to extend the tracheal puncture site. The tracheal opening is extended to $38 \mathrm{~F}$. The tracheal cannula is then inserted. Dilational tracheotomy is contraindicated in patients for whom primary intubation is difficult or who exhibit anatomical peculiarities (e.g., tracheomalacia, ankylosing spondylitis). Pathological changes in the cervical spine (e.g., unstable fractures) are also seen as contraindications. received

May 20, 2015

accepted after revision

September 15, 2015

published online

October 29, 2015
DOI http://dx.doi.org/

10.1055/s-0035-1566263. ISSN 2194-7635. (c) 2016 Georg Thieme Verlag KG
Stuttgart · New York

License terms

(c) $(1) \$$ 
Various complications like intra- and perioperative bleeding can occur. Pneumothorax and injury of the trachea are also possible. Tracheal stenosis may also occur as a late complication. ${ }^{2}$

The first change of cannula after dilational tracheotomy is not recommended until the seventh day after the procedure. It is assumed that a canal develops.

In the case described here, dislocation of the cannula on the ninth day after tracheotomy caused serious problems even though a straightforward cannula change had been performed two days previously.

\section{Case Report}

We present a case of a 70-year-old man who was severely ill. He had undergone amputation of the lower leg as a result of osteomyelitis of the tarsal bone with cellulitis. The patient had also been intubated and was dependent on mechanical ventilation because of bilateral pneumonia. It was not clear how long the patient would need ventilation, so an initial dilational tracheotomy was performed using the Ciaglia Blue Rhino technique (Cook Medical Inc., Bloomington, Indiana, United States). There were no reported problems. Ventilation functioned correctly thereafter. The thoracic X-ray (-Fig. 1) nevertheless showed an unusually stretched tracheal cannula with correct positioning of the cannula in the trachea; both lungs were ventilated to the same extent.

Nine days later, the tracheal cannula became dislocated during a repositioning maneuver. Recannulation of the trachea was no longer possible. Even with local extension of the incision it was not possible to locate the tracheal opening with certainty (-Fig. 2A). Only after additional blunt exploration did it appear likely that the entry point of the tracheal cannula was deep in the mediastinum. The attempt at recannulation was terminated and attempts were made to intubate the patient orally. This procedure proved extremely difficult because of a large swelling in the glottis area. At the same time it was necessary to reanimate the patient owing to bilateral pneumothorax. Thoracic drains were inserted on

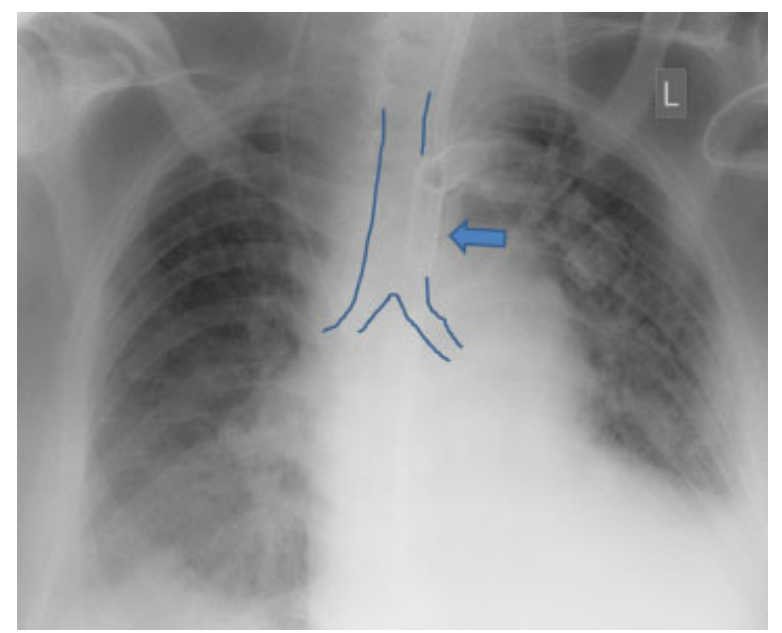

Fig. 1 Thoracic X-ray after dilational tracheotomy (using Blue Rhino technique). both sides. Several attempts at oral intubation were made before it succeeded. After starting ventilation, it was not possible to ventilate the patient sufficiently because all the air escaped through the tracheostomy. Adequate ventilation only became possible after the tracheotomy incision had been digitally closed. It was decided to advance the ventilation tube further and thus to bridge the tracheal leak. Bronchoscopy was then performed. This showed the position of the ventilation tube in the right main bronchus. After the tube had been withdrawn, a gaping lesion became apparent in the front wall of the trachea $\sim 1.5 \mathrm{~cm}$ above the main carina. The tube was then advanced into the right main bronchus once again and the patient was ventilated unilaterally. The situation was therefore very precarious. It appeared that the tracheostoma had been created too low down. We decided to attempt to seal the leak using cervical access before thoracotomy became necessary. The neck was stretched as far as possible in the operating theater. After sterile rinsing and covering of the operation area, the cervical incision was excised and prepared to a depth. It became apparent that the patient had a grade 3 goiter which had not previously been diagnosed. The very wide thyroid isthmus was severed between two clamps and sutured. It was then possible to see the full length of the trachea ( - Fig. 2B). With deep palpation of the mediastinum, a hole of the width of a finger could be felt in the front wall of the trachea. The hole began immediately below the brachiocephalic trunk which could be felt cranially to it ( - Fig. 2C). A video mediastinoscope was then used. This made it possible to inspect the tracheal defect ( $\mathbf{F i g}$. 3A). A gaping defect was visible taking up the entire front wall of the trachea. The trachea distal to this defect was subjected to blunt mobilization under direct video mediastinoscopic visualization. In this way, it was possible to mobilize the remaining trachea and both main bronchi. The distal trachea became so mobile that primary airtight closure of the tracheal defect using transverse sutures (PDS 4-0; - Fig. 3B) was possible. In addition, a conventional tracheotomy was performed approximately two cartilaginous rings down from the cricoid cartilage. The trachea was safely cannulated with a tracheal cannula (9.0 $\mathrm{CH}$ ). Ventilation could be performed without difficulty. The thoracic X-ray showed a correctly positioned tracheal cannula. Unfortunately, the process was discontinued 2 days later because a cranial CT scan showed diffuse hypoxic brain damage. The patient died on the same day exhibiting multiple organ failure.

\section{Discussion}

Dilational tracheotomy is a procedure of proven value and is performed frequently, particularly in intensive care units. Various techniques have been described. At this hospital, the Ciaglia Blue Rhino technique is performed especially often. Bronchoscopy is recommended for all dilational tracheotomy procedures. Puncturing the trachea in height of the second or third tracheal ring under visual control should make it possible to avoid injuring or perforating the rear wall. The trachea should also be punctured in the middle, vertically and not tangentially. In the case described above, the trachea 

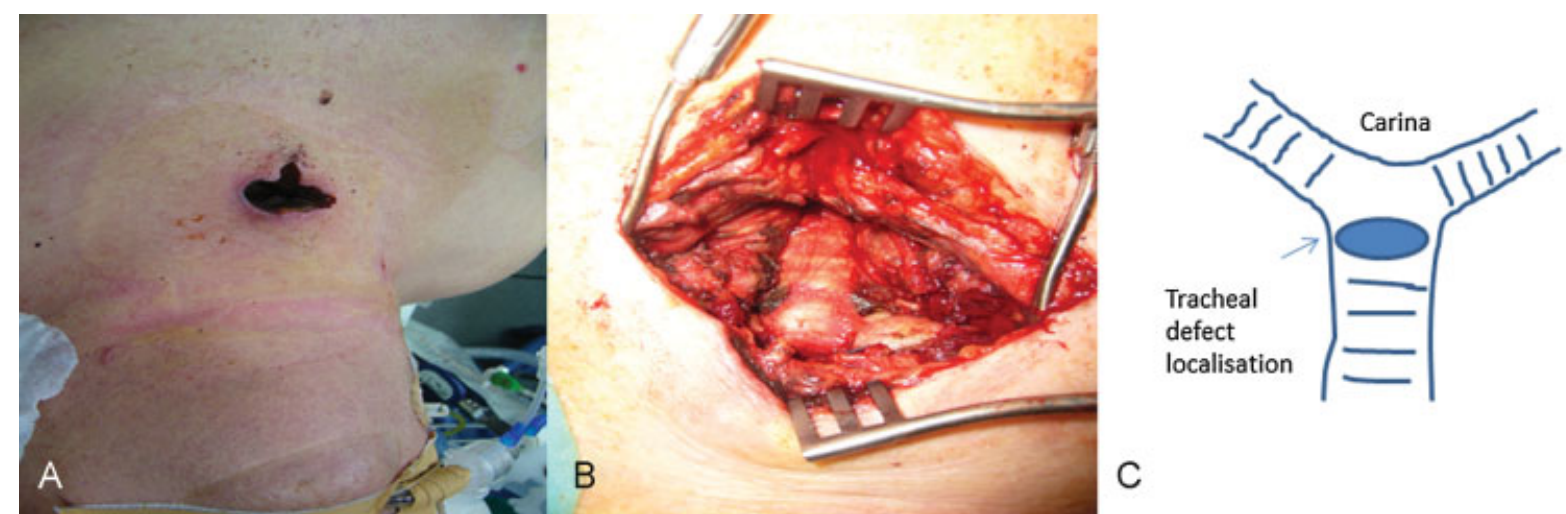

Fig. 2 (A) Throat site after unsuccessful attempt at recannulation. (B) Exposed cervical trachea. The point at which dilational tracheotomy was performed is not visible and is lower down in the mediastinum. (C) Schematic drawing of the situation in the trachea after dilational tracheotomy.

had probably been punctured unnoticed tangentially at its distal end. Because of the large goiter the puncture was unintentionally performed with a downward tilt. It was not possible to feel the enlarged thyroid gland before starting tracheotomy because the patient's neck was short. An enlarged thyroid is a contraindication for dilational tracheotomy. ${ }^{1}$ It is to be recommended that the soft tissue be examined using ultrasound in any patient with a short, thick neck so that those with an enlarged thyroid can be excluded. Tracheotomy should be performed open in these cases. It should be noted that the throat incision tends to be made too low down when the neck is short, thus increasing the risk that puncture will also be too low. In general, though, neck length does not affect the rate of complications associated with dilational tracheotomy. ${ }^{3}$ Unfortunately, the fact that the tracheal cannula was fitted too low down went unnoticed. When looking at the thoracic X-ray, the unusual stretching of the tracheal cannula could have been taken as an indication of incorrect positioning. However, as ventilation in this situation did not cause any problems, it is likely that no immediate consequences would have followed. We would have planned a cannula change with a Cook airway exchange catheter anyway, remaining ready to carry out oral intubation if necessary.
When the iatrogenic dislocation of the cannula happened, too much time was spent in trying to recannulate the trachea through the wound in the throat. Because of the low mediastinal insertion point and the additional goiter, it is not surprising that this was unsuccessful. It could not succeed, even with extension of the throat incision. To secure an airway, immediate oral intubation would have been the right step to take. Unfortunately, even oral intubation was made difficult by swelling of the larynx.

It was not possible to leave the tracheal defect caused by tracheotomy because ventilation with a normal tube did not appear possible due to the position above the main carina. Usually, closure is only possible with right-side thoracotomy. This would have endangered the patient because of his poor overall state. Our aim was therefore to try to seal the tracheal leak via the mediastinum. This does not require the patient to be repositioned. After exploration of the throat area and cutting of the wide thyroid isthmus, it became apparent that the trachea was not as short as had been thought. It seemed that we would be able to perform a conventional tracheotomy without problems. Because of our experiences with video mediastinoscopic release during resection of tracheal stenosis, its

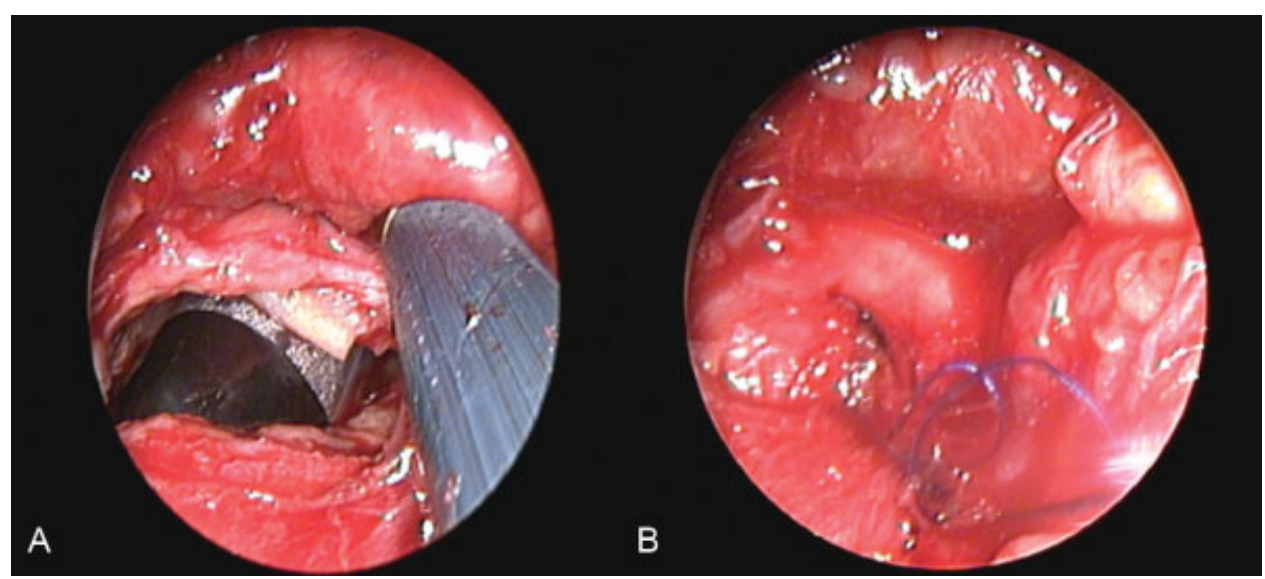

Fig. 3 Video mediastinoscopic image of the tracheal defect (A) after dilational tracheotomy and (B) after closure with sutures. 
use appeared obligatory in this situation. ${ }^{4}$ The distal trachea was mobilized $\sim 2$ to $3 \mathrm{~cm}$ in a cranial direction with visualization using video mediastinoscopy. This was far enough to create a secure transverse seal of the tracheal hole. This technique made it possible to avoid a complex thoracotomy.

The case described above shows clearly how important it is to inspect the local soft tissue of the throat beforehand. If a goiter is present, it cannot be palpated with certainty when the neck is short and thick. However, a thick neck per se is not a contraindication for dilational tracheotomy. ${ }^{5}$ Ultrasound examination of the throat is essential as a decision aid when choosing the appropriate tracheotomy technique. We would always choose open tracheotomy for a patient with a short neck and an enlarged thyroid.

It should also be noted that the opening created in PDT should be made at the same level as in conventional tracheotomy: that of the second or third tracheal ring. Our case shows just how fatal it can be when tracheotomy is performed too low down, especially when the tracheal cannula becomes dislocated. When puncturing the trachea, it is important to strike its lower part upright and not at an angle or tangentially. It is essential that the surgeon performing the tracheotomy checks the angle at which the puncture is performed. It is recommended that the trachea be punctured under bronchoscopic visualization. It is im- portant to strike the trachea in the middle and to avoid damaging the rear wall. Particular attention should be paid to the precise height of puncture. The endoscopist has a special role to play here.

Visualization using a video mediastinoscope makes secure transmediastinal closure possible in cases where a tracheostoma has been created too low down. In this way, thoracotomy can be avoided.

\section{References}

1 Koscielny S, Guntinas-Lichius O. Dilatation tracheotomy update : indications, limitations and management of complications [in German]. HNO 2009;57(12):1291-1300

2 Meininger D, Walcher F, Byhahn C. Tracheostomy in intensive care long-term ventilation : indications, techniques and complications [in German]. Chirurg 2011;82(2):107-110, 112-115

3 Tabaee A, Geng E, Lin J, et al. Impact of neck length on the safety of percutaneous and surgical tracheotomy: a prospective, randomized study. Laryngoscope 2005;115(9):1685-1690

4 Kirschbaum A, Teymoortash A. Video mediastinoscopically assisted tracheal release in treatment of long tracheal stenosis. Thorac Cardiovasc Surg Rep 2013;2(1):32-34

5 Rosseland LA, Laake JH, Stubhaug A. Percutaneous dilatational tracheotomy in intensive care unit patients with increased bleeding risk or obesity. A prospective analysis of 1000 procedures. Acta Anaesthesiol Scand 2011;55(7):835-841 